\title{
A Study of Testing Tools for the Fatigue Caused by the Stereoscopic Video Considering Temporal and Spatial Characteristics
}

\author{
Gil Ja So, Sang Hyun Kim, and Jeong Yeop Kim, Member, IACSIT
}

\begin{abstract}
The position of objects, the characteristics of the world in the content, the movement of objects, the color of object have to be considered to test viewer fatigue in the stereoscopic content. We studied what elements of the content can be factors which cause fatigue to viewer. We divide these factors into two groups, spatial or temporal factors and suggest the component for the tool testing viewer fatigue caused by stereoscopic contents.
\end{abstract}

Index Terms-Human fatigue for the stereoscopic contents, stereoscopy, OGRE 3D, tool of testing viewer fatigue.

\section{INTRODUCTION}

The reason which users feel fatigue as seeing stereoscopic movies is that stereoscopic image is created as the way that the human unify the differences between the two eyes to form the realistic illusion. Stereoscopic images are created as two images of the same scene from left eye and right eye. These two images are slightly different in the horizontal displacement. These two images then shown to the viewer by way of binocular arrangement.

The technology to present stereoscopic images to viewer have to consider the difference of the binocular disparity of the viewers and the ability of the unifying the two images in the brain.

Contents such as movies, animations, games can be produced with the stereoscopic technology [1]. However stereoscopic images are hard to get comfortable images. Many various directing techniques are used to increase the three-dimensional illusion for viewers. There needs to time for user to adapt their perception to the view when a fast object burst out from the screen to user throw the z-axis. The relationship of near, middle, far distance can be used to increase three-dimensional illusion [2] and [3]. The first person sight can give profound realism in the stereoscopic contents, like as movies, games. However, poor directing of contents make a user feel fatigue when see stereoscopic contents [4]. This is the reason to develop tool to test user's fatigue as building contents. Testing after production of

Manuscript received November 10, 2012; revised January 23, 2013.

Gil-Ja So is with the Department of Cyber Police and Science, University of Young-San, 288 Junam-ro, Yangsan-siKyungsangnam-do 626-790, Korea (e-mail: kjso@ysu.ac.kr).

Sang-Hyun Kim is with the Department of Computer Engineering, University of Young-San, 288 Junam-ro, Yangsan-siKyungsangnam-do 626-790, Korea (e-mail: ksh50@ysu.ac.kr).

Jeong-Yeop Kim is with the Department of Game Contents, University of Young-San, 99 Pilbong-gilHaeundae-Gu, Busan, 612-743, Korea (e-mail: neocopy@ysu.ac.kr). contents is complete is the most expensive. So, we need to develop a tool to test fatigue of viewer when see stereoscopic images.

Factors causing fatigue of viewer in stereoscopic contents can be divided into two, spatial factor and temporal factor. Temporal factors are horizontal or vertical movements, the velocity of movement, scene transitions, et al. Spatial factors are the amount of the number of objects which would make up the background in contents, the complexity of objects, and the nature of the texture of objects, color or brightness.

We describe a novel method for developing a tool to test the fatigue caused by stereoscopic image, considering temporal and spatial factor. The new method is implemented as an API extension for use with OGRE 3D, a 3D graphic engine usually used in game development. We have created two camera for left and right eye in the same scene with OGRE 3D.

\section{OGRE 3D}

OGRE3D is a 3D graphic engine to create real-time 3D applications with $\mathrm{C}++$. OGRE 3D support Direct 3D and OpenGL. The advantage of using this is highly customable, flexible scene management. OGRE 3D use predefined classes for scene organization if they suit or plug in user own subclass to gain full control over the scene organization[5]. We can insert plug in rendering stereoscopic image into the scene management. So, building the $3 \mathrm{~d}$ scene can follow custom ways without regarding to the rendering method, making two images for left and right eye, stereoscopic images.

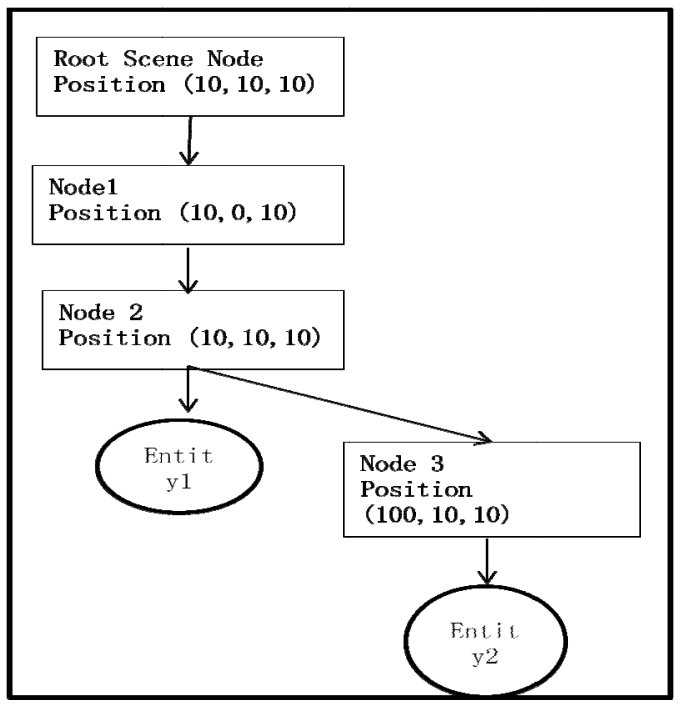

Fig. 1. An example of the scene graph. 
The most of classes of OGRE 3D engine take one role of 3 roles: Scene Management, Resource Management, Rendering. OGRE 3D can organize scenes hierarchically; nodes allow objects to be attached to each other and can move following each others. Resource Management can manage all resources needed for rendering like as geometry, textures, fonts, et al. The most important task for this is the loading, re-use and unloading of resources carefully. OGRE3D can get the visuals on the screen through the lower-level of the rendering pipeline.

Fig. 1 shows an example of the hierarchical scene graph.

The entity position is determined relatively to the parent of the node. In Fig. 1, the position of entity 1 and entity 2 will be $(30,20,30)$ and $(130,30,40)$ in $x, y, z$ coordinates. When we move the parent node 5 units along $y$-axis, all children will also be moved by 5 units along the $y$-axis [5] and [6].

\section{FACTORS CAUSING FATIGUE IN STEREOSCOPIC CONTENTS}

There are many researches which have studied about factors causing viewing fatigue or discomfort of viewers in stereoscopic contents. It has been known that big disparity is preferred for string 3D visual impact but often causes viewer fatigue. So, many researchers have tried to find a way to adjust disparity depending on viewer's condition[7]. However, elements of the content can cause viewer fatigue or discomfort, like as the amount of the objects in the content, colors or textures used in the object, fast or slow movement of the object, the interval time of two scenes, et al [8] and [9].

Table I shows the relationship between the element of the content and the viewer fatigue that can be caused by the element.

TABLE I: THE RELATIONSHIP BETWEEN THE ELEMENT OF THE CONTENT AND THE VIEWER FATIGUE

\begin{tabular}{|c|c|}
\hline $\begin{array}{l}\text { Element of the } \\
\text { content }\end{array}$ & $\begin{array}{c}\text { Characteristics of viewer fatigue caused by the } \\
\text { element }\end{array}$ \\
\hline Size of the world & Viewers feel cramped for space in the narrow space. \\
\hline $\begin{array}{l}\text { Velocity of the } \\
\text { object }\end{array}$ & Viewers feel dizzy when objects move fast. \\
\hline $\begin{array}{l}\text { Movement of the } \\
\text { camera }\end{array}$ & $\begin{array}{l}\text { Viewers feel dizzy when movement of the camera is } \\
\text { fast or the viewpoint of the camera changes too } \\
\text { often. }\end{array}$ \\
\hline $\begin{array}{l}\text { Displacement of } \\
\text { objects in the } \\
\text { world }\end{array}$ & $\begin{array}{l}\text { The distance of the nearest object and the most } \\
\text { distant object can increase the scope of the disparity } \\
\text { of viewer, which cause viewer fatigue in eyes. }\end{array}$ \\
\hline
\end{tabular}

We have divided these factors into two groups, temporal or spatial factor and implemented a tool to test viewer fatigue for various values of these factors. Temporal factors are the velocity of the object and spatial factors are the complexity of objects which are displaced in the world of the content and the color of the object.

\section{TOOLS FOR TESTING VIEWER FATIGUE}

\section{A. Overview of the Tool}

OGRE3D supports plug in for rendering stereoscopic images, the stereoscopy. The stereoscopy can obtain OGRE's forum. This plug-in support stereoscopic camera can render image for left and right eyes.

There are two modes: Anaglyph mode, interlaced mode. In the anaglyph mode, the image is made of a red and a cyan layer representing the left and the right eye. This can be viewed with simple red/cyan glasses. In the interlaced mode, the left and the right images are mixed with a check board or with a strips pattern. We have implemented a tool in the interlaced mode.

Fig. 2 is a diagram of the testing tool. The core sub-systems of the tool are menu manager and stereoscopic image render. Menu manager manipulate the input data of the viewer to find parameters to make them feel comfortable as seeing stereoscopic images.

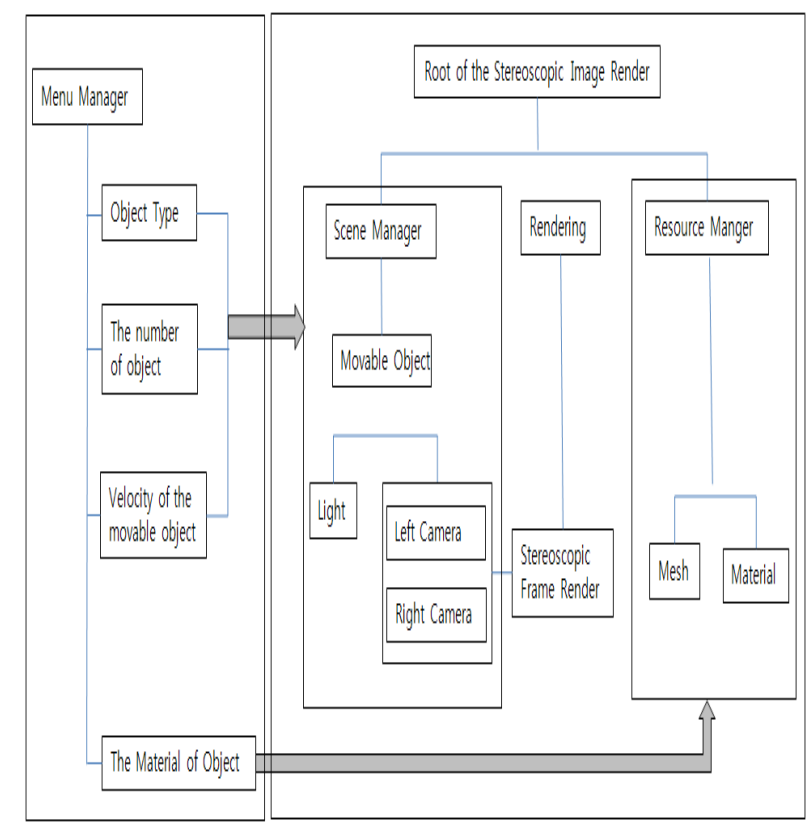

Fig. 2. Diagram of the testing tool.

Scene manager place objects to the number which viewer enter in menu and create left and right camera used in the stereoscopic frame render. Resource management changes the color of the object of which color is selected by the viewer. Velocity of the movable object can get the scene manager decide the position of object in the next frame.

\section{B. Testing Viewer Fatigue for the Spatial Factor}

Fig. 3 and Fig. 4 show the testing viewer fatigue for the complexity of the object, one of the spatial factors. Viewer can test their fatigue on the various values of the object type, the amount of the object. A tool support five object types, car, drum, robot, ball, house. These object type can be added to a tool by user. The number of objects range from 0 to 100 . Objects can be placed on the random position as to the number of objects which viewer would select.

Fig. 3 shows the testing viewer fatigue when objects are drum and the number of object is 8 . Objects are robots and the number of object is 20 in Fig. 4. The complexity of Fig. 4 is higher compared to Fig. 3. The amount of the viewer fatigue can be different as to viewer's conditions.

However, we can get the boundary which can cause fatigue to most viewers. 


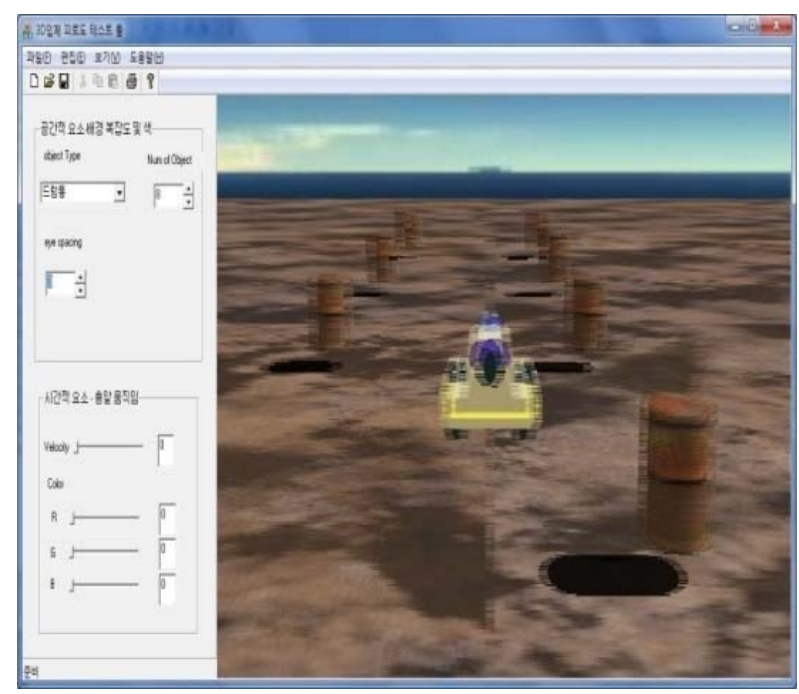

Fig. 3. Testing viewer fatigue for the complexity of the world I.

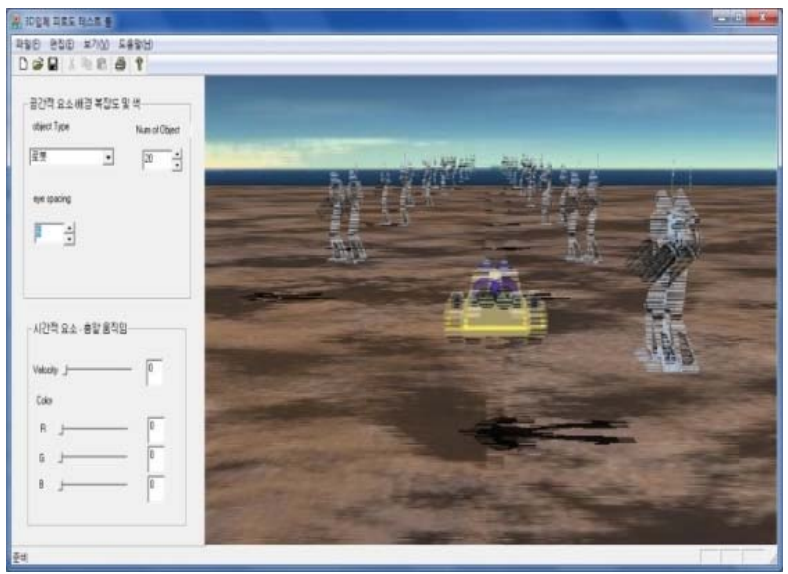

Fig. 4. Testing viewer fatigue for the complexity of the world II.

\section{Testing Viewer Fatigue for the Temporal Factor}

Fig. 4 shows the testing viewer fatigue for the temporal factor, depending on the various value of the velocity of the movement of the object in the content. Ball is used for the moving object in the Fig. 5. Moving object color can be changed by a viewer for testing their fatigue as seeing the stereoscopic scene. So, viewer can determine which color of object is more comfortable to them or the velocity of the object is less comfortable to them.

In Fig. 4, the color of the moving object, ball, is the red and the velocity is 50 .

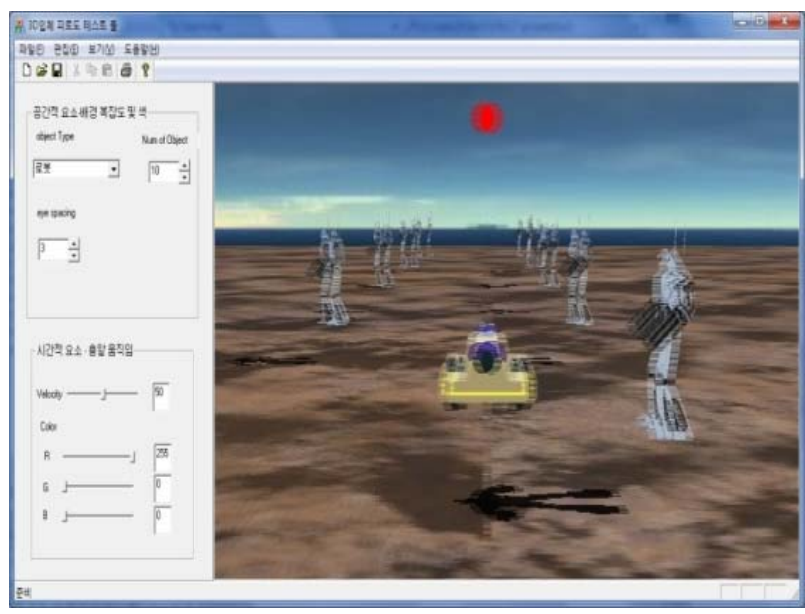

Fig. 5. Testing viewer fatigue for temporal factor.

\section{CONCLUSION}

Factors causing fatigue of viewer in stereoscopic contents can be divided into two, spatial factor and temporal factor. Temporal factors are horizontal or vertical movements, the velocity of movement, scene transitions, et al. Spatial factors are the amount of the number of objects which would make up the background in contents, the complexity of objects, and the nature of the texture of objects, color or brightness.

We describe a novel method for developing a tool to test the fatigue caused by stereoscopic image, considering temporal and spatial factor have implemented a tool in the interlaced mode.

\section{ACKNOWLEDGMENT}

This research is supported by Korea Creative Content Agency (KOCCA) in the Culture Technology(CT) Joint Research Center Project 2012.

\section{REFERENCES}

[1] C. W. Choi, "HUD factor classification of online FPS game for 3D stereoscopic application," M. S. thesis, Dept. of Media Broadcasting, Graduate School of Information Sciences, Soongsil Univ., Seoul, Korea, 2011

[2] N. G. Kim, "Human factors for 3D stereoscopic cinema," Communications of KIISE, vol. 29, no. 8, pp. 48-54, 2011.

[3] M. Lambooij and W. Ijsselsteijn, "Visual discomfort and visual fatigue of stereoscopic displays: A Review,” Imaging Sci. Technol., vol. 53, pp. 201-214, 2009

[4] C. Yuan, H. Pan, and S. Daly, "61.3: Stereoscopic 3D content depth tuning guided by human visual models," in Proc. SID Symposium Digest of Technical Papers, vol. 42, issue 1, June 2011. pp. 916-919

[5] G. Junker, "Pro OGRE 3D Programming (Expert's Voice in Open Source)," Springer Verlag, New York, 2006.

[6] Great looking 3D medical simulation made with Ogre3D. [Online]. Available: http://www.ogre3d.org

[7] H. Pan, C. Yuan, and S. Daly, "3D video disparity scaling for preference and prevention of discomfort," in Proc. SPIE 7863, Stereoscopic Displays and Applications XXII, 786306, Feb. 2011.

[8] K. Uki and P. A. Howarth, "Visual fatigue caused by viewing stereoscopic motion images: Background, theoriesand observations," Disp., vol. 29, pp. 106-116, 2008.

[9] S. H. Kim, M. J. Kim, and J. W. Kang, "An analysis of the visual characteristic of directing for three dimensional films," Journal of the Korea Contents Association, vol. 11, no. 2, pp. 229-237, 2011.

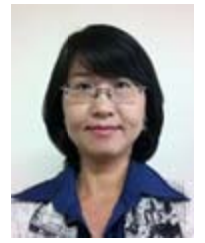

Gil Ja So received the Diploma in computer science from the Dong-eui University of Pusan, South Korea in 1994, and MS and $\mathrm{PhD}$ degrees, both in natural language processing of computer engineering, from the Pusan National university, South Korea in 1997 and 2012, respectively. From September 2001, she is with the Department of cyber police and science of the University of Young-San at Yangsan, Kyungnam as an assistant professor. Her doctoral dissertation work focused on the Korean Grammar Checker for parsing Korean documents and correcting context-sensitive words in documents. The topics she has worked on include virtual reality, game development and artificial intelligence of non-players of game, and 3D stereoscopic images. Her current research is on region-based intensity correction in 3D stereoscopic images. She is a member of the KMMS (Korea Multimedia Society) and KIPS (Korea Information Processing Society).

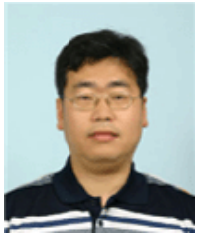

Sang Hyun Kim received the Diploma in electronics engineering from Kyung-Pook National University of Daegu, South Korea in 1991, and MS and PhD degrees, both in image processing of electronics engineering, from the same university, in 1993 and 1998, respectively. He was with the Samsung Medison Co. Ltd, in Korea as a senior researcher from 1998 to 2001. 
From September 2001, he is with the Department of Computer Engineering of the University of Young-San at Yangsan, Kyungnam as an associate professor. His doctoral dissertation work focused on the development of fractal image coding method and transmission for image processing, image communication and computer vision problems. The topics he has worked on include virtual reality, ultrasound image processing, medical image processing, image feature extraction and classification, and 3D stereoscopic images. His current research is on region-based intensity correction in 3D stereoscopic images. He is a member of the KMMS (Korea Multimedia Society) and KIPS (Korea Information Processing Society).

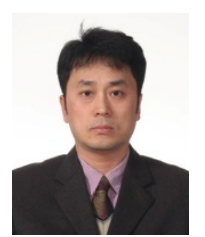

Jeong Yeop Kim received the Diploma in electronics engineering from Kyung-Pook National University of Daegu, South Korea in 1990, and MS and PhD degrees, both in image processing of electronics engineering, from the same university, in 1992 and 2000, respectively. He was with the Samsung Advanced Institute of Technology in Korea as a senior research staff from 1994 to 2000 . From March 2001, he is with the Department of Game Contents Science of the University of Young-San at Busan, as an associate professor. His doctoral dissertation work focused on detecting and adjusting the white point from single color image. The topics he has worked on include color printing, error diffusion, color correction and 3D stereoscopic images. His current research is on correction of color data in 3D stereoscopic images. $\mathrm{He}$ is a member of the KMMS (Korea Multimedia Society) and KIPS (Korea Information Processing Society). 